\title{
Scientist-Practitioner-Gap und Anwendungs- beobachtungen
}

\author{
Michael Linden \\ Forschungsgruppe Psychosomatische Rehabilitation, Charité Universitätsmedizin Berlin, Berlin, Deutschland
}

Im vorliegenden Heft geht es in einigen Beiträgen um Psychotherapie unter «Praxisbedingungen». Seit den Anfängen der evidenzbasierten Medizin gibt es eine Diskussion über das Verhältnis zwischen therapeutischer Praxis einerseits und Theorie bzw. wissenschaftlichen Therapiestudien andererseits. Sackett et al. [1996], die Doyens der evidenzbasierten Medizin, haben in einem Editorial unter dem Titel «Was evidenzbasierte Medizin ist und was nicht» dazu ausgeführt, dass evidenzbasierte Medizin die «gewissenhafte, explizite und verständige Anwendung der aktuell verfügbaren besten wissenschaftlichen Befunde bei Therapieentscheidungen in der Behandlung individueller Patienten» sei, d.h. eine Kombination sowohl aus individueller klinischer Expertise als auch aus externer Evidenz und explizit keine «Kochbuchmedizin». Evidenzbasierte Medizin ist damit also eigentlich ein Appell an die Intelligenz von Therapeuten.

In dem Versuch, zu bestimmen, was denn die «bestverfügbare Evidenz» ist, wurde aus evidenzbasierter Medizin häufig eine verbürokratisierte Leitlinienmedizin. Dies widerspricht eigentlich dem Wesen der evidenzbasierten Medizin im Sinne einer individualisierten Therapie. Zudem gibt es inzwischen auch eine umfangreiche wissenschaftliche Evidenz zu den Beschränkungen von Leitlinien - von der fehlenden Aktualität über eine unzureichende Validität und Probleme bei der Implementierung bis hin zu Negativfolgen bei der Umsetzung von Leitlinien.

Die Klärung, ob sich ein individueller Therapeut oder gar Gruppen von Therapeuten evidenzbasiert verhalten, erfolgt unter dem Stichwort der «Qualitätssicherung» üblicherweise durch versorgungsepidemiologische Untersuchungen im Sinne von «Anwendungsbeobachtungen». Auch dazu gibt es seit Langem umfangreiche methodische Diskussionen von Fachgesellschaften bis hin zu Bundesoberbehörden [Linden et al., 1994; BfArM und PEI, 2010]. Sie kamen dadurch auf, dass pharmazeutische Hersteller derartige Studien zum Qualitätsnachweis ihrer Produkte verwenden wollten. Im Ergebnis wurde fachlich und behördlich festgestellt, dass Anwendungsbeobachtungen geeignet sind, Erkenntnisse über das Therapeutenverhalten, die Therapieverläufe und gegebenenfalls über Nebenwirkungen zu generieren, aber keine Aussage über die Therapiewirksamkeit zulassen und daher auch nicht als Zulassungsstudien anerkannt werden. Die Ergebnisse dieser umfangreichen Diskussionen gelten grundsätzlich für jede Form der Versorgungsforschung und sollten auch allgemein bekannt sein.

Generell gilt, dass Praxisforschung die Untersuchung eines «Therapeutikums in der Hand eines Therapeuten» ist. Seit jeher gilt auch der Satz, dass jedes Kraut Gift und Heilmittel sein kann, abhängig von der Anwendung, d.h. vom Anwender. Dies gilt erst recht für die Psychotherapie. Was Anwendungsbeobachtungen leisten können und was nicht, kann beispielsweise der Arbeit von Hasenauer und Tschuschke [2017] in diesem Heft entnommen werden.

Im Sinne eines Qualitätsmonitorings erlauben Anwendungsbeobachtungen Aussagen zur «Therapieanwendung». Verfügbare Informationen zur in Behandlung aufgenommenen Population erlauben es, zu prüfen, ob es bezüglich der Zuweisungswege Verbesserungsansätze gibt. Auch werden Diagnoseprofile berichtet, was eventuell diagnostische Präferenzen erkennen lässt. Anhand der berichteten Zahl von Kurz- und Langzeitanträgen kann diskutiert werden, ob die avisierte Therapiedauer störungs-, ausbildungsoder richtlinienbegründet ist. Die Daten ermöglichen jedoch keine Qualitätsurteile, beispielsweise im Sinne eines «Scientist-Practitioner-Gap», mit Aussagen darüber, ob die beobachtete Therapieanwendung fachgerecht war oder nicht. Sie können nur Prüfaufträge begründen.

Anwendungsbeobachtungen erlauben auch explorative korrelative Berechnungen. So ist die Hauptfrage in der Publikation von Hasenauer und Tschuschke [2017] die nach der Korrelation verschiedener Kenngrößen mit dem Therapieergebnis zur Untersuchung von «Erfolgsprädiktoren in der kognitiven Verhaltenstherapie unter Praxisbedingungen». Korrelationen erlauben natürlich nie kausale Aussagen, können aber zu interessanten Hypothesen oder der Einleitung von Qualitätssicherungsmaßnahmen führen, wie im vorliegenden Fall: Hier stellte sich heraus, dass ausgerechnet Angststörungen ein schlechter Erfolgsprädiktor für Verhal-

\begin{tabular}{ll}
\hline KARGER & ( 2017 S. Karger Gm \\
& \\
$\begin{array}{l}\text { Information@Karger.com } \\
\text { www.karger.com }\end{array}$ & $\begin{array}{l}\text { Accessible online at: } \\
\text { www.karger.com/ver }\end{array}$
\end{tabular}


tenstherapie sind, was von den Autoren auch entsprechend diskutiert wird. Ebenso interessant ist die Vielzahl der sonstigen Parameter, die nicht mit dem Outcome korrelieren.

Natürlich erlauben Anwendungsbeobachtungen bei entsprechenden mehrzeitigen Erhebungen auch, die Frage nach dem Therapieverlauf zu stellen und Prä-Post-Unterschiede zu berichten. Dies kann allerdings nicht «belegen», dass eine therapeutische Wirksamkeit gegeben ist, sondern dies nur «nahelegen», wie die Autoren auch schreiben. Anwendungsbeobachtungen generieren immer eine Fülle von Daten, aus denen sich nie eine zwingende Interpretation ergibt. Wenn beispielsweise 36\% der Patienten eine Remission, 29\% eine partielle Besserung und 35\% keine Besserung oder sogar eine Verschlechterung zeigen, dann kann dies so interpretiert werden, dass die Daten überzeugende Therapieerfolge bei immerhin 36\% belegen oder dass die Therapie für eine nicht zufriedenstellende Besserung bei $64 \%$ verantwortlich ist. Die Interpretation von Beobachtungsdaten liegt im Belieben des Berichterstatters.

Was folgt aus diesen Überlegungen? Therapie unter Praxisbedingungen ist komplex, und die Normsetzung durch Leitlinien und ihre Überprüfung durch Anwendungsbeobachtungen ist wissenschaftlich sehr anspruchsvoll und verlangt dementsprechend methodisch wie in der Interpretation viel Sachkenntnis. Einfache Urteile oder Schlussfolgerungen sind grundsätzlich nicht zulässig. Aus Anwendungsbeobachtungen abgeleitete Qualitätsurteile, sowohl im Sinne eines Scientist-Practitioner-Gap als auch der Behauptung großer Wirkungen, sind selten fundiert und bedürfen stets einer methodenkritischen Kommentierung.

\section{Literatur}

Bundesinstitut für Arzneimittel und Medizinprodukte (BfArM) und Paul-Ehrlich-Institut für Impfstoffe und biomedizinische Arzneimittel (PEI): Empfehlungen des Bundesinstituts für Arzneimittel und Medizinprodukte und des Paul-Ehrlich-Instituts zur Planung, Durchführung und Auswertung von Anwendungsbeobachtungen vom 7. Juli 2010. www.bfarm.de/SharedDocs/ Bekanntmachungen/DE/Arzneimittel/klinPr/bm-KlinPr20100707-NichtinterventePr-pdf.pdf;jsessionid $=B 74063$ 70FE9EEOFAE49BE2CCEAF3ABA5.1_cid354?_blob= publicationFile $\mho v=6$ (letzter Abruf 5. Mai 2017).
Hasenauer M, Tschuschke V: Effektivität und Erfolgsprädiktoren in der kognitiven Verhaltenstherapie unter Praxisbedingungen. Verhaltenstherapie 2017;27:doi: $10.1159 / 000460824$

Linden M, Baier D, Beitinger H, Kohnen R, Osterheider M, Philipp M, Reimitz PE, Schaaf B, Weber HJ: Leitlinien zur Durchführung von Anwendungsbeobachtungen in der Psychopharmakotherapie. Nervenarzt 1994;65: 638-644.
Sackett DL, Rosenberg WMC, Gray JAM, Haynes RB, Richardson WS: Evidence based medicine: what it is und what it isn't. BMJ 1996;312:71-72. 